\title{
Rare TREM2 variants associated with Alzheimer's disease display reduced cell surface expression
}

\author{
Daniel W. Sirkis ${ }^{1 \dagger}$, Luke W. Bonham² ${ }^{\dagger}$, Renan E. Aparicio ${ }^{1}$, Ethan G. Geier ${ }^{2}$, Eliana Marisa Ramos ${ }^{3}$, Qing Wang ${ }^{3}$, \\ Anna Karydas², Zachary A. Miller², Bruce L. Miller², Giovanni Coppola ${ }^{3}$ and Jennifer S. Yokoyama ${ }^{2 *}$
}

\begin{abstract}
Rare variation in TREM2 has been associated with greater risk for Alzheimer's disease (AD). TREM2 encodes a cell surface receptor expressed on microglia and related cells, and the R47H variant associated with AD appears to affect the ability of TREM2 to bind extracellular ligands. In addition, other rare TREM2 mutations causing early-onset neurodegeneration are thought to impair cell surface expression. Using a sequence kernel association (SKAT) analysis in two independent AD cohorts, we found significant enrichment of rare TREM2 variants not previously characterized at the protein level. Heterologous expression of the identified variants showed that novel variants S31F and R47C displayed significantly reduced cell surface expression. In addition, we identified rare variant R136Q in a patient with language-predominant AD that also showed impaired surface expression. The results suggest rare TREM2 variants enriched in AD may be associated with altered TREM2 function and that AD risk may be conferred, in part, from altered TREM2 surface expression.
\end{abstract}

Keywords: TREM2, Genetics, Alzheimer's disease, Nasu-Hakola disease

Abbreviations: AD, Alzheimer's disease; ADSP, Alzheimer's disease sequencing project; CDR, Clinical dementia scale; DP, Read depth; ExAC, Exome Aggregation Consortium; GQ, Genotype quality score; IVPPA, Logopenic variant of primary progressive aphasia; MAF, Minor allele frequency; MMSE, Mini-mental state exam; NHD, Nasu-Hakola disease; SKAT, Sequence kernel association test; SNP, Single nucleotide polymorphism; UCSF MAC, University of California, San Francisco Memory and Aging Center; WES, Whole exome sequencing; WT, Wild type

\section{Introduction}

Alzheimer's disease (AD) is a common neurodegenerative disorder that occurs in older adults. Clinically, $\mathrm{AD}$ is characterized by a decline in cognitive function including memory, language, and/or visuospatial abilities. Aggregation of amyloid- $\beta$ and hyperphosphorylated tau, which result in the formation of plaques and neurofibrillary tangles, respectively, represent the pathological hallmarks of $\mathrm{AD}$. In addition to factors contributing to accumulation of amyloid and tau, changes in immune

\footnotetext{
* Correspondence: jennifer.yokoyama@ucsf.edu

${ }^{\dagger}$ Equal contributors

${ }^{2}$ Memory and Aging Center, UCSF, Department of Neurology, University of California, San Francisco, 675 Nelson Rising Lane, Suite 190, San Francisco, CA 94158, USA

Full list of author information is available at the end of the article
}

function resulting in increased inflammation are thought to contribute to disease pathogenesis and progression.

Common variants like $A P O E \& 4$ are the best characterized genetic risk factors associated with AD. However, rare genetic variation, which occurs at $<1 \%$ minor allele frequency (MAF) in a given population, is becoming increasingly appreciated for its contribution to neurodegenerative disease. These infrequent variants often have more potent biological effects and can occur in genes encoding proteins intimately linked to underlying protein pathology. Rare variants that confer both risk for [1-5] and protection from [6,7] different forms of neurodegeneration have been identified, but, due to their low MAF, most of these studies required very large cohorts to confirm the effect of these single variants on disease. 
TREM2 is a widely studied gene known to harbor rare variation that can either cause or contribute to risk for distinct neurodegenerative diseases. Homozygous or compound heterozygous mutations in TREM2 are known to cause Nasu-Hakola disease (NHD) or an early-onset frontotemporal dementia (FTD)-like syndrome, while rare variation in TREM2 increases risk for $\mathrm{AD}$, and may also increase risk for FTD, Parkinson's disease, and amyotrophic lateral sclerosis [8-10]. In the brain, TREM2 is an innate immune system receptor expressed primarily on microglia [11]. It has been implicated in sensing damage signals, promoting microglial survival, and regulating central nervous system inflammation [12-14]. In particular, the $\mathrm{R} 47 \mathrm{H}$ variant in TREM2 has been associated with $\mathrm{AD}$ risk in populations of European descent [4, 5], and is thought to alter microglial function $[13,15]$. Recent evidence suggests that the $\mathrm{R} 47 \mathrm{H}$ variant acts by altering TREM2's ability to bind lipoproteins and apolipoproteins, which may ultimately prevent microglia from efficiently absorbing amyloid- $\beta$-lipoprotein complexes [16].

Assessment of mutation burden can alleviate requirements for large cohorts by accounting for the overall risk contribution of rare and even unique variation observed in the same gene but in different individuals. Gene-based analysis offers the unique advantage of weighing the combined effects of multiple variants (common and/or rare) into a single statistical measure of disease risk $[17,18]$. Combining rare variants into a single analysis increases power to detect disease-associated risk in a gene using a relatively small cohort [17]. Furthermore, characterizing distinct rare variants occurring within the same functional domain of a particular protein may offer additional insight into shared pathogenic mechanisms. Of the available gene-based tests, the sequence kernel association test (SKAT) and its variants have proven reliable under multiple cohort sizes and have high mean power when compared to other tests [18-20].

In this study, we assessed deep sequencing data from over 150 genes previously linked to neurodegenerative, neuropsychiatric, and neurodevelopmental phenotypes for rare variant burden contributing to $\mathrm{AD}$. We confirmed that mutation burden in TREM2 is robustly associated with $\mathrm{AD}$ risk in two independent cohorts. We then characterized biochemically a subset of rare TREM2 variants to test whether they alter cell surface expression as a means of assessing their functional significance. Our analysis showed that several of the rare variants identified in $\mathrm{AD}$ indeed significantly reduced overall expression as well as cell surface expression of TREM2, suggesting that these variants may reduce protein function and contribute to disease risk.

\section{Materials and methods}

\section{Participants and clinical assessment}

For the discovery genetic analysis, 115 males and $161 \mathrm{fe}-$ males were evaluated at the University of California, San
Francisco Memory and Aging Center (UCSF MAC), and had genetic data available for analysis. All participants underwent clinical assessment with an in-person visit at the UCSF MAC that included a neurologic exam, cognitive assessment [21, 22] and medical history. Each participant's study partner was also interviewed regarding functional abilities. A multidisciplinary team composed of a neurologist, neuropsychologist, and nurse then established clinical diagnoses for cases according to consensus criteria for $A D$ and its subtypes [23, 24]. All healthy controls underwent a similar assessment, including study partner interview, and a consensus team of clinicians then established clinical diagnosis of cognitively normal. Controls in this study had Mini-Mental State Exam (MMSE) [25] scores $\geq 26$ or a Clinical Dementia Rating Scale (CDR) [26] of 0, no participant or informant report of cognitive concerns or decline in the prior year, and no evidence from clinical visit suggesting a neurodegenerative disorder (per team neurologist). Detailed demographic information is included in Table 1. Individuals harboring a known disease mutation or with a family history of neurodegeneration were excluded from the study.

Replication analysis was performed on samples from the case-control component of the Alzheimer's Disease Sequencing Project (ADSP), a Presidential Initiative established to identify new genes and alleles contributing to $\mathrm{AD}$ risk, $\mathrm{AD}$ protection, and targets for new $\mathrm{AD}$ therapies, particularly for late-onset AD. The discovery phase of this project generated whole exome sequencing (WES) data for 10,061 unrelated individuals $(N=5,096$ cases, $N=4,965$ controls) from the Alzheimer's Disease Genetics Consortium and the Cohorts for Heart Aging Research in Genomic Epidemiology consortia, of which 5,560 are included in the replication analysis (see Table 1 for cohort demographics). All cases met criteria for probable or definite AD based on clinical assessment, or had neuropathological features of AD upon brain autopsy. Pathological staging was made according to criteria set forth in Braak and Braak (1995) [27]. Cases received a Braak staging score greater than or equal to 3 . All controls were clinically assessed for dementia or had an absence of neuropathological AD features upon autopsy (Braak score of 2 or less). Individuals carrying a known disease mutation were excluded from the analyses. All sample phenotype and demographic data were obtained from dbGAP (study accession phs000572.v6.p4; table accession pht004306.v4.p4.c1).

All participants in both analyses were unrelated white individuals (confirmed by identity-by-descent testing in the replication analysis or self-described for those without GWAS data available). Non-Caucasian individuals were excluded due to the insufficient number of participants and potential for confounding background 
Table 1 Study participant characteristics

\begin{tabular}{|c|c|c|c|c|}
\hline Cohort & Variable & $A D$ & Control & $P$-value \\
\hline \multirow[t]{8}{*}{ Discovery (UCSF) } & $\mathrm{N}$ & 31 & 245 & \\
\hline & Age at Onset / First Visit & $77.8 \pm 4.5$ & $68.5 \pm 8.5$ & $p<0.001$ \\
\hline & $\operatorname{Sex}(M / F)$ & $16 / 15$ & $99 / 146$ & 0.18 \\
\hline & Edu (Years, Mean \pm SD) & $17.0 \pm 3.7$ & $17.3 \pm 2.1$ & 0.45 \\
\hline & CDR $($ Mean \pm SD) & $0.8 \pm 0.3$ & $0.0 \pm 0.1$ & $p<0.001$ \\
\hline & MMSE (Mean \pm SD) & $22.2 \pm 5.3$ & $29.4 \pm 0.8$ & $p<0.001$ \\
\hline & APOE $\varepsilon 4$ dose $(0 / 1 / 2)$ & $9 / 17 / 5$ & $190 / 48 / 4$ & $p<0.001$ \\
\hline & \# Pathological Confirmed AD & 12 & & \\
\hline \multirow[t]{8}{*}{ Replication (ADSP) } & $\mathrm{N}$ & 2927 & 2633 & \\
\hline & Age at Onset / First Visit & $75.3 \pm 8.4$ & $85.5 \pm 5.1$ & $p<0.001$ \\
\hline & $\operatorname{Sex}(M / F)$ & $1299 / 1628$ & $1185 / 1448$ & 0.639334 \\
\hline & Edu (Years, Mean \pm SD) & NA & NA & NA \\
\hline & CDR $($ Mean \pm SD) & NA & NA & NA \\
\hline & MMSE (Mean \pm SD) & NA & NA & NA \\
\hline & APOE $\varepsilon 4$ dose $(0 / 1$ / 2) & 1660 / 1184 / 83 & 2239 / 386 / 8 & $p<0.001$ \\
\hline & \# Pathological Confirmed AD & 1057 & & \\
\hline
\end{tabular}

Summary demographic, clinical, and genetic information is shown for the Discovery and Replication Cohorts. Note: three individuals in the UCSF Cohort (all controls) do not currently have APOE \&4 genotyped. M Male, F Female, Edu Education, SD Standard Deviation, MMSE Mini Mental State Examination, NA

Not Available

genetics. All aspects of the study were approved by the UCSF Institutional Review Board and written informed consent was obtained from all participants and surrogates (as per UCSF Institutional Review Board protocol).

\section{Sequencing}

The UCSF cohort was screened using targeted sequencing of more than 150 RefSeq genes previously implicated in neurodegenerative dementia, including the most common causative genes for Mendelian forms of AD and FTD. Exonic regions for these genes were captured using a custom-designed Nimblegen SeqCap EZ Choice (Roche) library and sequenced on an Illumina HiSeq2500 at the UCLA Neuroscience Genomics Core (Los Angeles, CA). Sequence reads were mapped to the GRCh37/hg19 reference genome and variants were interactively joint-called with GATK according to GATK Best Practices recommendations (https://www.broadinstitute.org/gatk/ [28]).

ADSP samples underwent WES at one of three NHGRI funded large-scale sequencing centers at Baylor, the Broad Institute, or Washington University. Whole exome capture was performed using either the Illumina Rapid Capture Exome kit or VCRome v2.1 kit (Nimblegen), and paired-end reads were generated using an Illumina HiSeq 2000. Sequence reads were aligned to the GRCh37 reference genome using the Burrows-Wheeler aligner [29], and variants were jointly called across the entire cohort using Atlas V2 software (Baylor) or GATK (Broad). Variants underwent pipeline-specific quality control prior to merging the variants that were concordant between the two sets of variants. The ADSP also performed initial quality control checks on sample information, phenotypes, and genotype data to ensure that these data were of high quality and suitable for downstream analysis.

\section{Quality control and post-processing}

After joint-calling, variants were filtered according to previously established criteria [30]. Briefly, we kept jointcalled variants with genotype quality (GQ) scores greater than 30 and read depth (DP) scores greater than 20. The resulting file was annotated with gene names using the Variant Effect Predictor in Ensembl. The predicted effect of each variant were determined using PolyPhen and SIFT. Prior to analysis, we used PLINK [31] to remove individuals with genotyping rates below $95 \%$, SNPs (single nucleotide polymorphisms) with genotyping rates below 95, and SNPs with a MAF greater than $1 \%$. Gene SNP sets were created from exonic SNPs classified as missense and nonsense variants. For our replication analysis, we created SNP sets using the same genes that were available for study in our discovery cohort.

\section{Genetic analyses}

Following previously published criteria [18], we limited our analyses to gene SNP sets with 4 or more SNPs available. For our discovery analysis we conducted a SKAT analysis in the amnestic AD cohort from UCSF. Our replication analysis in the ADSP amnestic AD cohort used the same testing parameters and techniques. We repeated the aforementioned analysis in subset of 
the ADSP cohort that had pathologically confirmed AD. Finally, to test whether rare variation in TREM2 is associated with clinically heterogeneous $\mathrm{AD}$, we ran a SKAT analysis in a subset of the cohort which included amnestic $\mathrm{AD}$, early-onset $\mathrm{AD}$, executive (frontal) $\mathrm{AD}$, and the logopenic variant of primary progressive aphasia (lvPPA).

\section{Antibodies}

The HA.11 monoclonal antibody used to detect HATREM2 was from Covance, and the clathrin heavy chain monoclonal antibody was from BD Transduction Laboratories.

\section{Molecular biology}

The human TREM2 cDNA was obtained from R\&D Systems, amplified by PCR and inserted into the pEGFPN1 vector after first removing the EGFP coding sequence. To facilitate detection of TREM2, an HA epitope tag and linker sequence identical to that used in Kleinberger et al. [14], were inserted after the TREM2 signal peptide using the Phusion high-fidelity DNA polymerase (NEB) system for site-directed mutagenesis. All TREM2 variants were similarly generated using Phusion, with the HA-TREM2 construct serving as template DNA. All constructs were verified by sequencing at the UC Berkeley DNA Sequencing Facility.

\section{Cell culture}

HEK-293T cells were maintained at the UC Berkeley Cell Culture Facility under standard conditions. Cells were transiently transfected using Lipofectamine 2000 (ThermoFisher) according to the manufacturer's instructions. Culture medium was typically changed $4 \mathrm{~h}$ after transfection, and experiments were carried out the following day.

\section{Immunoblotting}

Cells were harvested on ice by washing with cold PBS followed by lysing in a buffer containing $100 \mathrm{mM} \mathrm{NaCl}$, $10 \mathrm{mM}$ Tris-Cl, $\mathrm{pH}$ 7.6, 1 \% (v/v) Triton X-100 and Complete protease inhibitor cocktail (Roche). Tritoninsoluble material was sedimented by centrifugation at $20,000 \mathrm{~g}$ for $10 \mathrm{~min}$ at $4{ }^{\circ} \mathrm{C}$. Supernatants were mixed with 5X SDS-PAGE sample buffer supplemented with $\mathrm{DTT}$, then heated at $55{ }^{\circ} \mathrm{C}$ for $10 \mathrm{~min}$ prior to running in $4-20 \%$ acrylamide gradient gels (Life Technologies and Bio-Rad). After SDS-PAGE, proteins were transferred onto PVDF membranes (EMD Millipore), blocked in $5 \%$ non-fat milk (dissolved in PBS containing $0.1 \%$ Tween-20), and probed with $\mathrm{HA}$ and $\mathrm{CHC}$ antibodies at 1:2,500 and 1:10,000, respectively. Blots were developed using enhanced chemiluminescence and imaged on a ChemiDoc digital imager (Bio-Rad). Protein signals were quantified using ImageJ (NIH). For overall TREM2 expression analysis, the TREM2 signals derived from cell lysates were first normalized to the corresponding $\mathrm{CHC}$ signal, then calculated as a fraction of the WT signal.

\section{Cell surface biotinylation}

Cell surface biotinylation was carried out in a manner similar to that performed in Kleinberger et al., 2014. Briefly, cells were washed at room temperature (RT) with PBS and labeled with the EZ-Link Sulfo-NHS-SSBiotin reagent (ThermoFisher) at $1 \mathrm{mg} / \mathrm{ml}$ in PBS for $15 \mathrm{~min}$. Cells were then placed on ice, washed with cold Tris-buffered saline to quench the biotin reagent, then washed with cold PBS and finally lysed and clarified as described above. To capture biotinylated proteins, StrepTactin resin (iba) was added to the clarified lysates and the mixtures rotated at $4{ }^{\circ} \mathrm{C}$ for $1 \mathrm{~h}$. The resin was then pelleted and washed multiple times with lysis buffer. Finally, 2X SDS-PAGE sample buffer supplemented with DTT was added to the washed resin, and the samples were vortexed, heated and prepared for immunoblotting as described above. For the analysis of surface-labeled TREM2, we quantified the entire surface-labeled signal (including mature and immature bands) by densitometry and normalized the signal of individual variants to the WT signal.

\section{Statistical analysis}

We used the "SKAT" package [18] in $\mathrm{R}$ to conduct all gene-based association tests. The SKAT package allows users to conduct sequence kernel association tests, which are powerful when a portion of the variants in a region are noncausal or variant effects are in different directions. All genetic analyses using in the MAC and ADSP cohorts were completed using R.

Protein expression analyses and plots were completed using Graphpad Prism 6 (La Jolla, CA). Protein expression differences were established with ANOVA tests. We used the Holm-Sidak method for our post hoc testing.

\section{Results \\ Cohort descriptions}

Two hundred and seventy six individuals (245 healthy controls and 31 individuals with AD) participated in this study's discovery analysis; 5,560 (2,633 healthy controls and 2,927 individuals with AD) participated in this study's replication analysis. Detailed cohort characteristics by diagnostic grouping for each cohort are provided in Table 1. In both cohorts, there were significant differences in age by diagnostic grouping and $A P O E \& 4$ dosage, and no significant differences by sex. As expected, in the discovery cohort, there were significant differences in mean CDR and MMSE score. There was no significant difference in education in the discovery cohort. 
Rare variation in TREM2 is enriched in amnestic Alzheimer's disease

\section{Discovery analysis}

After quality control and annotation, 157 gene SNP sets were identified. The identified genes are listed in Additional file 1: List S1. Of these, 43 gene SNP sets had 4 or more eligible SNPs and were included in the aggregate burden analysis. The most significant gene SNP set in our discovery analysis was TREM2 ( $p=$ 0.001). After multiple testing correction using the Bonferroni method, the p-value for TREM2 was 0.04. Summary data for this analysis are shown in Table 2. The variants included in the TREM2 SNP set and their amino acid coding changes are summarized in
Table 3. Full results for all 43 SNP sets are shown in Additional file 1: Table S1.

\section{Replication analysis in clinically diagnosed and pathologically confirmed AD}

We started with the same 157 genes available in the discovery analysis. Of these, 65 gene SNP sets had 4 or more eligible SNPs. The most significant gene SNP set in the replication cohort was also TREM2 $\left(p=2.88 \times 10^{-4}\right)$. After multiple testing correction using the Bonferroni method, the p-value for TREM2 was 0.02. The variants included in the TREM2 SNP set and their amino acid coding changes are summarized in Table 3. There were 1,057 neuropathologically confirmed AD cases in the ADSP cohort and we

Table 2 Aggregate variant burden analysis in discovery and replication cohorts

\begin{tabular}{|c|c|c|c|c|c|c|}
\hline Cohort & Gene & Testable SNPs & SNPs tested & MAC & $P$-value & Corrected $P$-value \\
\hline \multirow[t]{10}{*}{ Discovery (UCSF) } & TREM2 & 19 & 8 & 10 & $1.00 \times 10^{-3}$ & 0.04 \\
\hline & SMG6 & 38 & 4 & 4 & $3.42 \times 10^{-2}$ & NS \\
\hline & ARHGAP27 & 17 & 4 & 6 & 0.1 & NS \\
\hline & HSPA6 & 18 & 6 & 9 & 0.15 & NS \\
\hline & LRRK2 & 46 & 9 & 10 & 0.16 & NS \\
\hline & WDR81 & 28 & 10 & 12 & 0.2 & NS \\
\hline & PLCD3 & 16 & 4 & 5 & 0.2 & NS \\
\hline & MAP1B & 28 & 7 & 9 & 0.22 & NS \\
\hline & UBAP1 & 11 & 4 & 4 & 0.22 & NS \\
\hline & NPEPPS & 11 & 4 & 6 & 0.23 & NS \\
\hline \multirow[t]{10}{*}{ Replication (ADSP) } & TREM2 & 41 & 24 & 330 & $2.88 \times 10^{-4}$ & 0.02 \\
\hline & GYPC & 24 & 16 & 31 & $5.19 \times 10^{-3}$ & NS \\
\hline & UBAP2 & 123 & 72 & 207 & 0.05 & NS \\
\hline & PACRG & 20 & 14 & 93 & 0.06 & NS \\
\hline & ZNF621 & 32 & 17 & 24 & 0.06 & NS \\
\hline & $E P H X 2$ & 56 & 38 & 284 & 0.08 & NS \\
\hline & RPIA & 28 & 9 & 11 & 0.09 & NS \\
\hline & FYN & 28 & 13 & 43 & 0.1 & NS \\
\hline & HSPA6 & 25 & 20 & 89 & 0.11 & NS \\
\hline & HSPA4 & 59 & 28 & 183 & 0.12 & NS \\
\hline \multirow[t]{10}{*}{ Replication (ADSP - Pathology Confirmed) } & TREM2 & 41 & 16 & 192 & $2.11 \times 10^{-4}$ & 0.01 \\
\hline & CLU & 51 & 19 & 108 & $2.78 \times 10^{-3}$ & NS \\
\hline & KIF24 & 136 & 54 & 243 & $6.00 \times 10^{-3}$ & NS \\
\hline & GYPC & 24 & 8 & 12 & 0.02 & NS \\
\hline & BIN1 & 61 & 16 & 41 & 0.03 & NS \\
\hline & RNF19A & 56 & 23 & 48 & 0.03 & NS \\
\hline & MR1 & 43 & 18 & 106 & 0.04 & NS \\
\hline & $E P H X 2$ & 56 & 29 & 181 & 0.06 & NS \\
\hline & RPIA & 28 & 7 & 9 & 0.09 & NS \\
\hline & $P A C R G$ & 20 & 12 & 58 & 0.09 & NS \\
\hline
\end{tabular}


Table 3 TREM2 SNP-set Characteristics

\begin{tabular}{|c|c|c|c|c|c|c|c|}
\hline Cohort & $\mathrm{CHR}$ & $\mathrm{BP}$ & SNP & Minor Allele (+/- strand) & AD MAF & Control MAF & AA change \\
\hline \multirow[t]{8}{*}{ Discovery (UCSF) } & 6 & 41126395 & - & $\mathrm{A} / \mathrm{T}$ & 0 & 0.002041 & E202D \\
\hline & 6 & 41126619 & rs138355759 & $\mathrm{A} / \mathrm{T}$ & 0 & 0.002041 & T223I \\
\hline & 6 & 41126801 & rs371702633 & $C / G$ & 0 & 0.002041 & S162R \\
\hline & 6 & 41127605 & rs149622783 & T/A & 0 & 0.002041 & R136Q \\
\hline & 6 & 41127606 & - & $\mathrm{A} / \mathrm{T}$ & 0 & 0.002041 & R136W \\
\hline & 6 & 41129004 & - & $\mathrm{A} / \mathrm{T}$ & 0 & 0.002041 & A130S \\
\hline & 6 & 41129133 & rs142232675 & T/A & 0 & 0.002041 & $\mathrm{D} 87 \mathrm{~N}$ \\
\hline & 6 & 41129252 & rs75932628 & $\mathrm{T} / \mathrm{A}$ & 0.04839 & 0 & $\mathrm{R} 47 \mathrm{H}$ \\
\hline \multirow[t]{14}{*}{ Replication (ADSP) } & 6 & 41126642 & - & $C / G$ & 0.000171 & 0 & H215Q \\
\hline & 6 & 41126701 & - & T/A & 0.000171 & 0 & A196T \\
\hline & 6 & 41126801 & rs371702633 & $C / G$ & 0.000342 & 0 & S162R \\
\hline & 6 & 41127543 & rs2234255 & $\mathrm{A} / \mathrm{T}$ & 0.00103 & 0 & H157Y \\
\hline & 6 & 41127561 & rs79011726 & T/A & 0.000172 & 0 & E151K \\
\hline & 6 & 41129133 & rs142232675 & T/A & 0.001708 & 0.0009498 & $\mathrm{D} 87 \mathrm{~N}$ \\
\hline & 6 & 41129195 & rs201258663 & $\mathrm{A} / \mathrm{T}$ & 0.000171 & 0 & T66M \\
\hline & 6 & 41129252 & rs75932628 & T/A & 0.008944 & 0.001716 & R47H \\
\hline & 6 & 41129253 & rs753325601 & $\mathrm{A} / \mathrm{T}$ & 0.000172 & 0 & R47C \\
\hline & 6 & 41129295 & rs104894002 & $\mathrm{A} / \mathrm{T}$ & 0.000345 & 0 & Q33* \\
\hline & 6 & 41129300 & rs746216516 & $\mathrm{A} / \mathrm{T}$ & 0.000173 & 0 & S31F \\
\hline & 6 & 41129309 & rs2234252 & $\mathrm{A} / \mathrm{T}$ & 0.000173 & 0 & $\mathrm{~A} 28 \mathrm{~V}$ \\
\hline & 6 & 41129313 & rs768745050 & $\mathrm{T} / \mathrm{A}$ & 0.000173 & 0 & V27M \\
\hline & 6 & 41129345 & rs 777808487 & $\mathrm{~A} / \mathrm{T}$ & 0.000177 & 0 & $\mathrm{~S} 16 \mathrm{~F}$ \\
\hline
\end{tabular}

Detailed results for TREM2 SNP sets used in discovery and replication analyses. Variants in bold were included in the protein expression experiments. $C H R$ Chromosome, BP Base Pair, rsID Reference SNP Cluster ID, MAF Minor Allele Frequency, AA Amino Acid

performed an additional analysis restricted to these cases versus all available ADSP controls. Of the 157 genes from the discovery analysis, 61 gene SNP sets had 4 or more eligible SNPs. TREM2 was also the most significant gene SNP set in the pathology-confirmed AD replication analysis $\left(p=2.11 \times 10^{-4}\right)$. After multiple testing correction using the Bonferroni method, the p-value for TREM2 was 0.01 . Summary data for both the clinically diagnosed $A D$ replication cohort and pathologically diagnosed $\mathrm{AD}$ cohort are shown in Table 2. Full results for all 65 gene SNP sets in the clinically diagnosed $\mathrm{AD}$ cohort are shown in Additional file 1: Table S2. Full results for all 61 gene SNP sets in the pathologically diagnosed AD cohort are shown in Additional file 1: Table S3. Together, these results confirm our initial findings from the discovery analysis that rare variants in the exons of TREM2 are enriched in amnestic AD.

\section{Secondary analysis in clinically heterogeneous AD}

Following our discovery and replication analyses, we conducted an exploratory analysis to identify rare variants in TREM2 in four clinical variants of AD. We limited our analysis to UCSF participants. The aggregate burden $\mathrm{p}$-value for TREM2 across all AD subtypes was 0.044 . Of note, in this analysis we identified an additional R136Q mutation in an individual with lvPPA. In total, we thus identified R136Q in one patient with lvPPA and in one control in the initial discovery analysis of amnestic AD. A summary of all variants discovered in our analyses by diagnostic grouping and cohort is presented in Additional file 1: Table S4.

\section{Rare TREM2 variants show altered cell surface expression}

To characterize the rare TREM2 variants identified in the UCSF cohort at the protein level, we performed sitedirected mutagenesis on a human TREM 2 cDNA. We restricted our analysis to coding changes affecting the canonical splice variant of TREM2. For the discovery cohort analyses in amnestic and heterogeneous $\mathrm{AD}$, we generated seven point variants, including those we identified in amnestic $A D(R 47 H)$, atypical $A D /$ lvPPA and controls (R136Q) as well as those identified specifically in controls (D87N, A130S, R136W, S162R and T223I). As an internal control for our analyses, we also generated the Y38C variant involved in early-onset frontotemporal dementia (FTD) [32], which is known to be 
defective for protein maturation. These variants were transfected into HEK-293T cells and their expression analyzed by immunoblotting. All variants other than Y38C showed apparently normal protein maturation (Fig. 1a). Interestingly, the immature bands of variants R136Q and T223I showed slightly altered migration by SDS-PAGE, with R136Q migrating slightly slower and T223I slightly faster. Although variant R136Q occasionally showed accumulation of its immature form (Fig. 1a), we did not observe this effect consistently (Fig. 1b). Because we identified R136Q in a patient with atypical AD (as well as in one control), and variants R136Q and R136W have both been observed in AD cases in other studies $[2,4,5]$, we further characterized these variants. Of note, R136W has been suggested in unpublished work [33] to show reduced cell surface expression. Thus, we performed cell surface biotinylation on cells expressing these variants. We observed a modest but statistically significant reduction in surface expression for variant R136Q (Fig. 1b, c). In addition, we observed an even larger defect for R136W, highlighting the importance of residue Arg 136 for normal TREM2 surface expression levels. Analysis of the overall expression level of these variants in whole-cell lysates indicated that
R136W was significantly reduced (Fig. 1b, c). Mutation of residue Arg 136 thus appears capable of altering both cell surface and overall TREM2 expression.

We next characterized selected variants identified in the replication cohort, all of which were identified in $\mathrm{AD}$ cases. We focused on variants localizing to the extracellular domain, as this is the region affected by the $\mathrm{R} 47 \mathrm{H}$ variant as well as many of the NHD-causing mutations in TREM2. As with the initial set of variants characterized, we observed apparently normal protein maturation in the five variants studied (V27M, A28V, S31F, R47C and E151K; Fig. 2a, left). However, we observed significant reductions in overall expression as well as cell surface expression for variants S31F and R47C (Fig. 2a, b). Variant E151K showed reduced overall expression, but its trend toward reduced surface expression did not reach significance. To our knowledge, variant S31F has not been previously reported as a risk variant in $\mathrm{AD}$ and thus is a novel TREM2 variant for the field. Variant R47C also appears to be a novel variant and was found in a patient with pathologically confirmed AD (Braak stage 6). Interestingly, $\mathrm{R} 47 \mathrm{C}$ affects the same arginine residue altered in the $\mathrm{R} 47 \mathrm{H}$ variant. Finally, variant A28V showed a significant increase in cell surface expression.

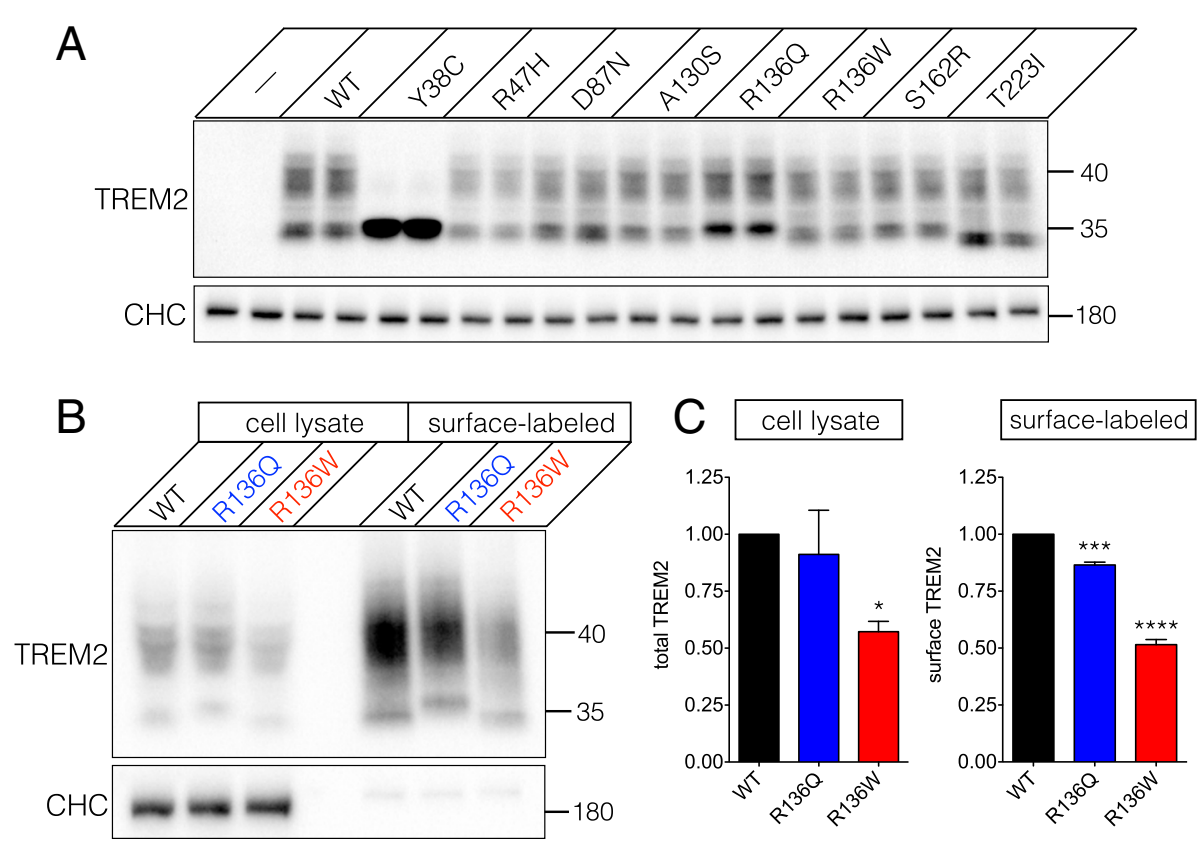

Fig. 1 Biochemical characterization of rare TREM2 variants identified at the UCSF Memory and Aging Center. a Seven rare TREM2 variants were transiently expressed in HEK-293T cells and compared to cells expressing wild type (WT) TREM2, the Y38C variant or untransfected cells (-). One day after transfection, cells were lysed and the lysates analyzed (in duplicate) by immunoblotting for TREM2 to assess expression, maturation, and electrophoretic mobility. The newly identified variants showed apparently normal maturation, but variant R136Q, identified in a patient with atypical AD, showed slower migration of the immature band. Clathrin heavy chain (CHC) was used as a loading control. b and $\mathbf{c}$ Whole-cell lysate and cell surface biotinylation analysis for variants R136Q and R136W demonstrate significantly reduced overall expression for variant R136W and significantly reduced surface expression for both variants ( $^{*}<0.05$, *** $p<0.001$, *** $p<0.0001$ by ANOVA followed by Holm-Sidak post hoc test). CHC was used a loading control for the cell lysates and to confirm the lack of non-specific biotinylation of cytosolic proteins. Results were quantified from three independent experiments 


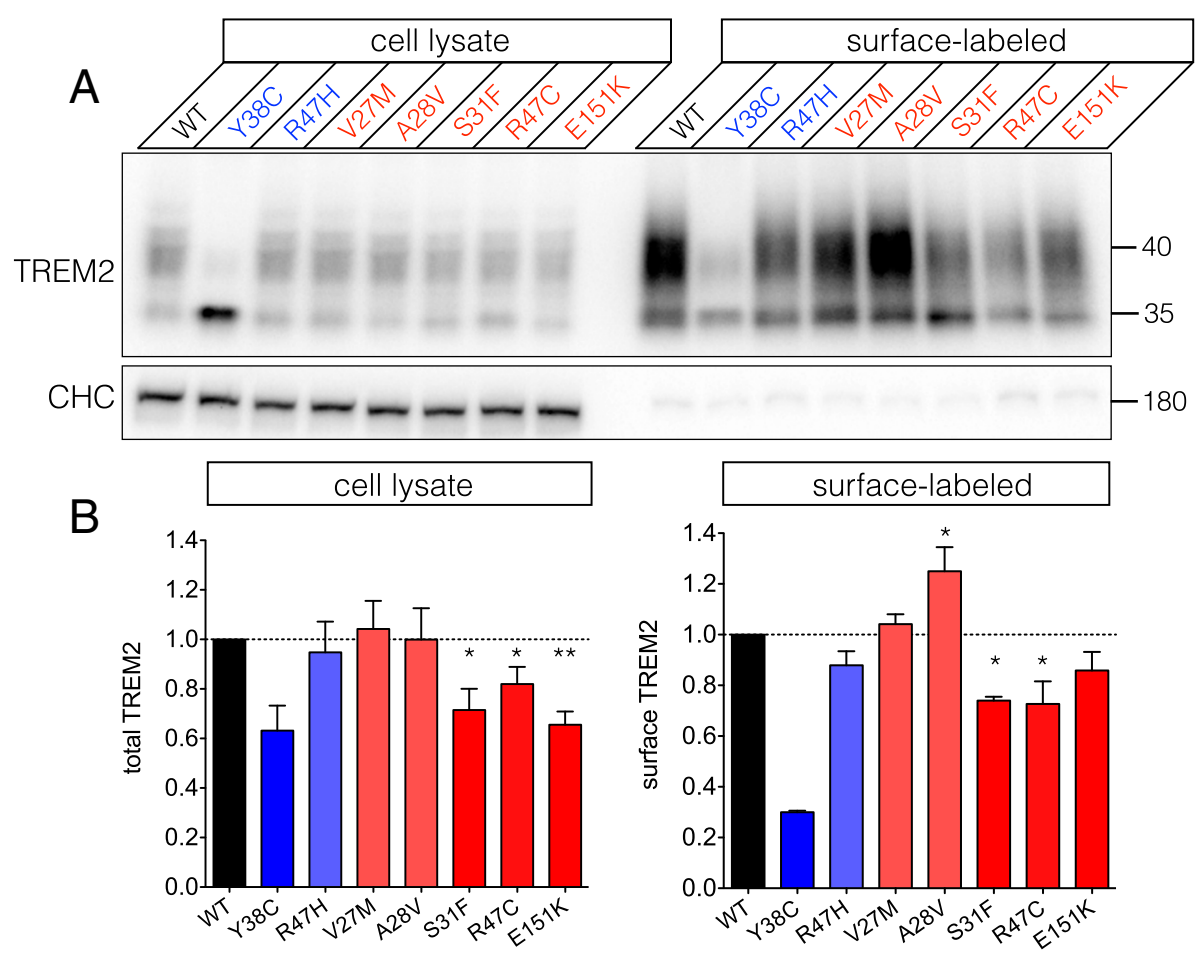

Fig. 2 Biochemical analysis of additional rare TREM2 variants identified in the Alzheimer's Disease Sequencing Project (ADSP) replication cohort. $\mathbf{a}$ and $\mathbf{b}$ Five additional rare TREM2 variants identified in patients with AD were analyzed as in Fig. 1. All variants showed normal maturation and mobility by immunoblot analysis. CHC was used as a loading control for the cell lysates and to control for non-specific biotinylation (as above). Whole-cell lysate and cell surface biotinylation analysis indicated that variants S31F, R47C and E151K showed significantly reduced overall expression, while S31F and R47C showed significantly reduced surface expression. Variant A28V specifically showed increased surface expression. Variants Y38C and R47H were used, respectively, as internal controls for severe or modest reductions in cell surface expression $\left({ }^{*} p<0.05,{ }^{* *} p<0.01\right.$ by ANOVA followed by Holm-Sidak post hoc test). Results were quantified from 3-4 independent experiments for each variant

\section{Discussion}

We confirmed association of aggregate rare variation in TREM2 with AD in two independent cohorts, including in a subset of individuals with pathologically confirmed AD. Two of the variants identified in AD, S31F and R47C, have not, to our knowledge, been described before. In addition, the R136Q variant identified in an atypical form of $\mathrm{AD}$ has not been previously characterized at the protein level. Using heterologous expression, we found that these three variants show a significant reduction in cell surface expression relative to WT TREM2.

Rare homozygous or compound heterozygous mutations in TREM2 cause NHD or an early-onset FTD syndrome without bone involvement $[8,34]$. These include missense mutations such as $\mathrm{Y} 38 \mathrm{C}, \mathrm{T} 66 \mathrm{M}$ and D86V that occur within the Ig-like domain of TREM2 [32, 35, 36]. Variants $\mathrm{Y} 38 \mathrm{C}$ and T66M have been shown to have impaired cell surface expression [14, 37], and we now demonstrate that the novel variants S31F and R47C, which also localize to the Ig-like domain, show reduced surface expression. Thus, it is possible that modestly reduced TREM2 cell surface expression in heterozygotes increases risk for late-onset neurodegeneration, while severely reduced surface expression in homozygotes leads to early-onset FTD or NHD. By extension, we hypothesize that homozygous carriers of S31F, R47C or $\mathrm{R} 136 \mathrm{Q}$, if identified, might be at greater risk for AD neurodegeneration, relative to heterozygotes. In contrast to the above variants, variant $\mathrm{A} 28 \mathrm{~V}$, which was also identified in an AD case, showed significantly increased surface expression. It is thus currently unclear if this variant is impaired in another way (e.g., defective ligand binding) or if it contributes risk for disease.

In all of our surface expression analyses, we observed that the immature form of TREM2 was capable of reaching the cell surface. Although this was reported previously for disease-causing variants $\mathrm{Y} 38 \mathrm{C}$ and $\mathrm{T} 66 \mathrm{M}$, it was not observed for the WT protein [14]. We speculate that this discrepancy may be due to the different expression systems used (transient expression in this paper vs. stable expression in [14]). Importantly, however, we confirmed the strong reduction in surface expression for variant $\mathrm{Y} 38 \mathrm{C}$ that was reported previously in Kleinberger et al. [14] and Park et al. [37], indicating the suitability of our expression method for cell surface labeling. 
TREM2 variant $\mathrm{R} 136 \mathrm{Q}$ was identified in one patient with a language-predominant form of $\mathrm{AD}$, the logopenic variant of primary progressive aphasia (lvPPA, [24]). We also identified R136Q and R136W in one control each, underscoring the point that these variants do not appear to be causative for disease. However, others have previously reported both of these variants in amnestic $A D$ $[2,5]$, and they have MAFs of 0.001278 and 0.0001381 , respectively ( 2 observations in 1,564 alleles and 1 observations in 7,240 alleles, respectively) in the Exome Aggregation Consortium (ExAC) database [38]. The reported MAF of $\mathrm{R} 47 \mathrm{H}$ is about 20 -fold greater than that reported for R136Q and is consistent with our observation of only one case harboring this variant.

We utilized an aggregate variant burden test implemented in the SKAT program to assess the effects of variation across multiple genes-including TREM2-on risk for $\mathrm{AD}$. Advantages of this package include that it makes no assumption about the direction or magnitude of an effect and its ability to account for both a large fraction of noncausal variants and causal variant effects that are in different directions. Some limitations occur when the number of SNPs required in each set results in exclusion of candidate genes. Analysis of larger cohorts with deep resequencing data will be required to expand coverage of rare variation across more genes.

Our finding that some variants do not alter cell-surface expression does not preclude these variants from altering $\mathrm{AD}$ risk via other mechanisms. For instance, the V27M and E151K variants did not show significantly reduced surface expression, but may be defective for ligand binding, as has been shown recently for $\mathrm{R} 47 \mathrm{H}$ and other variants $[16,39,40]$. Variant A28V, identified in an AD case and showing increased surface expression, may increase risk for disease by adversely affecting ligand binding, or, alternatively, may not affect risk for disease. Future functional studies such as lipoprotein binding and uptake assays will be required to further characterize the effects of the identified variants. We also identified several variants in controls that will require further genetic and functional characterization to determine whether they are likely to alter disease risk. For example, the D87N variant identified in both cases and controls in our cohorts, has recently been shown to display a defect in ligand binding [16] and may thus represent an AD risk variant.

Our study benefits from the analysis of multiple cohorts representing both amnestic and atypical forms of $\mathrm{AD}$, pathological confirmation in a subset of individuals from the replication cohort, and the ability to assess biochemically the effect of select variants on protein expression and cell surface expression. Caveats of the study include a limited number of patients in the discovery cohort-particularly of atypical AD syndromes-and, as mentioned above, the limited scope of genes analyzed.

\section{Conclusions}

In summary, we find that rare variation in TREM2, including two variants within the extracellular Ig-like domain, may be associated with risk for AD. Our findings further suggest that impaired overall and cell surface expression of TREM2 may contribute to risk for AD. In addition, since the well-known, AD-associated variant $\mathrm{R} 47 \mathrm{H}$ has been proposed to impair TREM2's ability to bind extracellular ligands $[12,39,40]$, it will be interesting to determine in the future whether the variants identified here similarly affect ligand binding. Variants that reduce surface expression without directly impairing ligand binding would be attractive targets for therapeutic intervention that focuses on restoring TREM2 expression at the cell surface.

\section{Additional file}

Additional file 1: Table S1. Full Results for Discovery Analysis. Table S2. Full Results for Clinically Diagnosed AD Replication Analysis. Table S3. Full Results for Pathologically Diagnosed AD Replication Analysis. Table S4. TREM2 Variants Categorized by Cohort and Phenotype. List S1. Genes Available in Discovery Analysis. Acknowledgment statement for the ADSP. (DOCX $49 \mathrm{~kb}$ )

\section{Acknowledgements}

We thank contributors who collected samples used in this study, as well as patients and their families, whose help and participation made this work possible.

\section{Funding}

Primary support for data analyses was provided by the NIA F32 AG050404 (DWS), NIA K01 AG049152 (JSY), Larry L. Hillblom Foundation 2012-A-015-FEL and 2016-A-005-SUP (JSY), and AFTD Susan Marcus Memorial Fund Clinical Research Grant (JSY). Additional support, including for assembly of cohorts, was provided by Hillblom Aging Network (BLM), NIA P50 AG023501 (BLM), and P01 AG1972403 (BLM), the John Douglas French Alzheimer's Foundation (GC), the Tau Consortium, and Takeda Pharmaceutical Company Limited. We acknowledge the support of the NINDS Informatics Center for Neurogenetics and Neurogenomics (P30 NS062691). ADSP data was obtained from dbGaP for study accession phs000572.v6.p4 and table accession pht004306.v4.p4.c1. Full funding acknowledgements for ADSP are provided in the Additional file 1.

\section{Availability of supporting data and materials}

UCSF MAC data will not be publicly shared. Investigators who wish to access UCSF MAC data must apply and be approved. Please see http://

memory.ucsf.edu/resources/data/ for more details. ADSP data will not be publicly shared. Investigators who wish to use ADSP data must apply. Please see https://www.niagads.org/adsp/ for more details.

\section{Authors' contributions}

DWS designed and performed the protein expression experiments, interpreted the data, and wrote the manuscript; LWB designed and performed the genetic analyses, interpreted the data, and wrote the manuscript; REA designed the TREM2 CDNA constructs; EGG designed and performed the genetic analyses and interpreted the data; EMR assisted with genotyping and interpretation of genetic data; QW prepared libraries for genotyping; AK participated in sample coordination and interpretation of results; ZAM conducted chart reviews to confirm patient phenotypes and participated in interpretation of results; BLM participated in sample coordination and interpretation of results; GC participated in sample coordination and genotyping and helped to draft the manuscript; JSY conceived the study, interpreted the data, and wrote the manuscript. All authors read and approved the final manuscript. 


\section{Authors' information}

Not applicable.

\section{Competing interests}

Takeda Pharmaceutical Company Limited funded genotyping for UCSF MAC participants, but played no role in the design of the study, interpretation of results, or preparation of the manuscript. The authors declare no competing interests.

\section{Consent for publication}

Not applicable.

\section{Ethical approval and consent to participate}

All aspects of the study were approved by the UCSF Institutional Review Board and written informed consent was obtained from all participants and surrogates (as per UCSF Institutional Review Board protocol).

\section{Author details}

'Department of Molecular and Cell Biology, Howard Hughes Medical Institute, University of California, Berkeley, Berkeley, CA 94720, USA. ${ }^{2}$ Memory and Aging Center, UCSF, Department of Neurology, University of California, San Francisco, 675 Nelson Rising Lane, Suite 190, San Francisco, CA 94158, USA. ${ }^{3}$ Department of Psychiatry and Semel Institute for Neuroscience and Human Behavior, The David Geffen School of Medicine at University of California Los Angeles, Los Angeles, CA 90095, USA.

\section{Received: 15 July 2016 Accepted: 17 August 2016}

\section{Published online: 02 September 2016}

\section{References}

1. Piccio L, Deming Y, Del JL, Laura Á, Holtzman DM, Fagan AM, et al. Cerebrospinal fluid soluble TREM2 is higher in Alzheimer disease and associated with mutation status. Acta Neuropathol. 2016;131:925-33.

2. Jin SC, Benitez BA, Karch CM, Cooper B, Skorupa T, Carrell D, et al. Coding variants in TREM2 increase risk for Alzheimer's disease. Hum Mol Genet. 2014;23:5838-46.

3. Coppola G, Chinnathambi S, Lee JJ, Dombroski BA, Baker MC, Soto-Ortolaza $\mathrm{Al}$, et al. Evidence for a role of the rare p.A152T variant in MAPT in increasing the risk for FTD-spectrum and Alzheimer's diseases. Hum Mol Genet. 2012;21:3500-12.

4. Jonsson T, Stefansson H, Steinberg S, Jonsdottir I, Jonsson PV, Snaedal J, et al. Variant of TREM2 associated with the risk of Alzheimer's disease. N Engl J Med. 2013;368:107-16

5. Guerreiro R, Wojtas A, Bras J, Carrasquillo M, Rogaeva E, Majounie E, et al. TREM2 variants in Alzheimer's disease. N Engl J Med. 2013;368:117-27.

6. Jonsson T, Atwal JK, Steinberg S, Snaedal J, Jonsson PV, Bjornsson S, et al. A mutation in APP protects against Alzheimer's disease and age-related cognitive decline. Nature. 2012:488:1-4.

7. Mead S, Whitfield J, Poulter M, Shah P, Uphill J, Campbell T, et al. A novel protective prion protein variant that colocalizes with kuru exposure. N Engl J Med. 2009;361:2056-65.

8. Painter MM, Atagi Y, Liu C-C, Rademakers $R, X u H$, Fryer JD, et al. TREM2 in CNS homeostasis and neurodegenerative disease. Mol Neurodegener. 2015;10:1-10.

9. Borroni B, Ferrari F, Galimberti D, Nacmias B, Barone C, Bagnoli S, et al. Heterozygous TREM2 mutations in frontotemporal dementia. Neurobiol Aging. 2014;35:7-10

10. Kiialainen A, Hovanes K, Paloneva J, Kopra O, Peltonen L. Dap12 and Trem2, molecules involved in innate immunity and neurodegeneration, are coexpressed in the CNS. Neurobiol Dis. 2005;18:314-22.

11. Zhang Y, Chen K, Sloan SA, Bennett ML, Scholze AR, O'Keeffe S, et al. An RNA-sequencing transcriptome and splicing database of glia, neurons, and vascular cells of the cerebral cortex. J Neurosci. 2014:34:11929-47.

12. Wang $Y$, Cella M, Mallinson $K$, Ulrich JD, Young KL, Robinette ML, et al. TREM2 lipid sensing sustains the microglial response in an Alzheimer's disease model. Cell. 2015;160:1061-71.

13. Colonna M, Wang Y. TREM2 variants: new keys to decipher Alzheimer disease pathogenesis. Nat Rev Neurosci. 2016;17:201-7.

14. Kleinberger G, Yamanishi $Y$, Suárez-Calvet M, Czirr E, Lohmann E, Cuyvers E, et al. TREM2 mutations implicated in neurodegeneration impair cell surface transport and phagocytosis. Sci Transl Med. 2014;6:1-12.
15. Yuan P, Condello C, Keene CD, Wang Y, Bird TD, Paul SM, et al. TREM2 haplodeficiency in mice and humans impairs the microglia barrier function leading to decreased amyloid compaction and severe axonal dystrophy. Neuron. 2016:90:724-39.

16. Yeh FL, Wang Y, Tom I, Gonzalez LC, Sheng M. TREM2 binds to apolipoproteins, including APOE and CLU/APOJ, and thereby facilitates uptake of amyloid-beta by microglia. Neuron. 2016;91:328-40.

17. Neale BM, Sham PC. The future of association studies: gene-based analysis and replication. Am J Hum Genet. 2004;75:353-62.

18. Lee S, Emond MJ, Bamshad MJ, Barnes KC, Rieder MJ, Nickerson DA, et al. Optimal unified approach for rare-variant association testing with application to small-sample case-control whole-exome sequencing studies. Am J Hum Genet. 2012:91:224-37.

19. Wu MC, Lee S, Cai T, Li Y, Boehnke M, Lin X. Rare-variant association testing for sequencing data with the sequence kernel association test. Am J Hum Genet. 2011;89:82-93.

20. Moutsianas L, Agarwala V, Fuchsberger C, Flannick J, Rivas MA, Gaulton KJ et al. The power of gene-based rare variant methods to detect diseaseassociated variation and test hypotheses about complex disease. PLoS Genet. 2015;11:e1005165.

21. Miller ZA, Mandelli ML, Rankin KP, Henry ML, Babiak MC, Frazier DT, et al. Handedness and language learning disability differentially distribute in progressive aphasia variants. Brain. 2013;136:3461-73.

22. Rankin KP, Kramer J, Miller BL. Patterns of cognitive and emotional empathy in frontotemporal lobar degeneration. Cogn Behav Neurol. 2005:18:28-36

23. Dubois B, Feldman $H$, Jacova C. Advancing research diagnostic criteria for Alzheimer's disease: the IWG-2 criteria. Lancet Neurol. 2014;13:614-29.

24. Gorno-Tempini ML, Hillis AE, Weintraub S, Kertesz A, Mendez M, Cappa SF, et al. Classification of primary progressive aphasia and its variants. Neurology. 2011;76:1006-14.

25. Folstein MF, Folstein SE, McHugh PR. "Mini-mental state". A practical method for grading the cognitive state of patients for the clinician. J Psychiatr Res. 1975;12:189-98.

26. Morris JC. The Clinical Dementia Rating (CDR): current version and scoring rules. Neurology. 1993:43:2412-4.

27. Braak H, Braak E. Neuropathological stageing of Alzheimer-related changes. Acta Neuropathol. 1991;82:239-59.

28. McKenna A, Hanna M, Banks E, Sivachenko A, Cibulskis K, Kernytsky A, et al. The genome analysis toolkit: a MapReduce framework for analyzing nextgeneration DNA sequencing data. Genome Res. 2010;20:1297-303.

29. Li H, Durbin R. Fast and accurate short read alignment with BurrowsWheeler transform. Bioinformatics. 2009;25:1754-60.

30. Carson AR, Smith EN, Matsui H, Brækkan SK, Jepsen K, Hansen J-B, et al. Effective filtering strategies to improve data quality from populationbased whole exome sequencing studies. BMC Bioinformatics. 2014;15:1-15.

31. Purcell S, Neale B, Todd-Brown K, Thomas L, Ferreira MA, Bender D, et al. PLINK: a tool set for whole-genome association and population-based linkage analyses. Am J Hum Genet. 2007:81:559-75.

32. Guerreiro R, Bilgic B, Guven G, Brás J, Rohrer J, Lohmann E, et al. A novel compound heterozygous mutation in TREM2 found in a Turkish frontotemporal dementia-like family. Neurobiol Aging. 2013;34:2890.

33. Jin SC. Identification of functional variants in Alzheimer's disease-associated genes. Louis: Washington University in St; 2014.

34. Paloneva J, Manninen T, Christman G, Hovanes K, Mandelin J, Adolfsson R, et al. Mutations in two genes encoding different subunits of a receptor signaling complex result in an identical disease phenotype. Am J Hum Genet. 2002;71:656-62.

35. Klunemann HH, Ridha BH, Magy L, Wherrett JR, Hemelsoet DM, Keen RW, et al. The genetic causes of basal ganglia calcification, dementia, and bone cysts: DAP12 and TREM2. Neurology. 2005;64:1502-7.

36. Guerreiro RJ, Lohmann E, Brás JM, Gibbs JR, Rohrer JD, Gurunlian N, et al. Using exome sequencing to reveal mutations in TREM2 presenting as a frontotemporal dementia-like syndrome without bone involvement. JAMA Neurol. 2013;70:78-84.

37. Park JS, Ji IJ, An HJ, Kang MJ, Kang SW, Kim DH, et al. Disease-Associated Mutations of TREM2 Alter the Processing of N-Linked Oligosaccharides in the Golgi Apparatus. Traffic. 2015;16:510-8.

38. Lek M, Karczewski K, Minikel E, Samocha K, Banks E, Fennell T, et al. Analysis of protein-coding genetic variation in 60,706 humans. bioRxiv. 2015. 
39. Atagi Y, Liu C-C, Painter MM, Chen X-F, Verbeeck C, Zheng H, et al. Apolipoprotein $\mathrm{E}$ is a ligand for triggering receptor expressed on myeloid cells 2 (TREM2). J Biol Chem. 2015;290:26043-50.

40. Bailey CC, DeVaux LB, Farzan M. The triggering receptor expressed on myeloid cells 2 binds apolipoprotein E. J Biol Chem. 2015;290: 26033-42.

Submit your next manuscript to BioMed Central and we will help you at every step:

- We accept pre-submission inquiries

- Our selector tool helps you to find the most relevant journal

- We provide round the clock customer support

- Convenient online submission

- Thorough peer review

- Inclusion in PubMed and all major indexing services

- Maximum visibility for your research

Submit your manuscript at www.biomedcentral.com/submit 